\title{
EIGENVALUES OF THE LAPLACIAN ON REGULAR POLYGONS AND POLYGONS RESULTING FROM THEIR DISECTION
}

\author{
L. M. Cureton \\ U.S. Department of Justice, 10th and Constitution Avenue, Washington, \\ DC 20530, U.S.A.
}

AND

\section{J. R. KutTLER}

The Johns Hopkins University, Applied Physics Laboratory, Johns Hopkins Road, Laurel, MD 20723-6099, U.S.A.

(Received 13 November 1997, and in final form 14 August 1998)

The eigenvalue problem is considered for the Laplacian on regular polygons, with either Dirichlet or Neumann boundary conditions, which will be related to the unit circle by a conformal mapping. The polygonal problem is then equivalent to a weighted eigenvalue problem on the circle with the same boundary conditions. Upper bounds are found for the eigenvalues by the Rayleigh-Ritz method, where the trial functions are the eigenfunctions of the unweighted problem on the circle. These are products of Bessel and trigonometrical functions, and so the required integrals simplify greatly, with a new recursion formula used to generate some Bessel function integrals. Numerical results are given for the case of the hexagon with Dirichlet conditions. Consideration of symmetry classes makes computations more efficient, and gives as a byproduct the eigenvalues of a number of polygons, such as trapezoids and diamonds, which result from disecting the hexagon. Comparisons of the hexagon results are made with previous work.

(C) 1999 Academic Press

\section{INTRODUCTION}

The eigenvalue problem is considered for the Laplacian

$$
\Delta \Phi+\lambda \Phi=0 \quad \text { on } R
$$

It arises from separating the time variable out of the wave equation, and so occurs in many applications. When $R$ is a bounded region of the plane, equation (1) may represent the propagation of a wave down a waveguide (electrical or acoustical), with uniform cross-section $R$. The eigenvalue $\lambda=k^{2}$, where $k$ is a cut-off frequency, and $\Phi$ is a mode of propagation. Equation (1) may also represent the vibration of an elastic membrane in the shape of $R$, with $k$ proportional to a vibrational frequency, and $\Phi$ in the shape of a vibrational mode. When $R$ is a 
polygon, with boundary $\partial R$ consisting of straight line segments, equation (1) may also represent the vibration of plates in the shape of $R$.

The boundary conditions considered are the Dirichlet condition

$$
\Phi=0 \quad \text { on } \partial R
$$

or the Neumann condition

$$
\frac{\partial \Phi}{\partial n}=0 \quad \text { on } \partial R
$$

The Dirichlet condition which governs TM-propagation in an electrical waveguide, is called a soft boundary in an acoustical waveguide, and governs fixed membranes and simply-supported plates. The Neumann condition which governs TE-propagation in electrical waveguides, is called a hard boundary in an acoustical waveguide, and governs free membranes.

Regular polygons will be considered, which will be related to the unit circle by a conformal mapping. Equation (1) is then equivalent to a weighted eigenvalue problem on the circle with the same boundary conditions [1]. Upper bounds are found for the eigenvalues by the Rayleigh-Ritz method [2], where the trial functions are the eigenfunctions of the unweighted problem on the circle. These are products of Bessel and trigonometrical functions, and so the required integrals simplify greatly, with a new recursion formula used to generate some Bessel function integrals.

The notion of using conformal mapping on a membrane/waveguide problem to obtain a numerically more tractible problem is not new. It was used with good effect in reference [3] to find the cut-off frequencies of a ridged waveguide. However, it is a very useful method which does not seem to be employed as much as it might be. The power of the method will be illustrated by using a well-known conformal map which takes the circle to a regular polygon.

The regular polygons are a canonical and classical set of membrane/waveguide shapes of enduring interest. The eigenvalues of equilateral triangles and squares can be found analytically, but the eigenvalues of regular polygons with more than four sides cannot, and even very accurate numerical approximations for the lowest eigenvalues seem scarce. Irie et al. [4] gave results accurate to about three significant figures for the first 14 eigenvalues of fixed regular polygons with three through ten sides. Molinari [5] used the same conformal map that we do to get rather rough estimates on the lowest eigenvalue of free regular polygonal membranes of sides five, six and eight.

Numerical results will be given for the case of the hexagon with Dirichlet conditions. This is the higher polygon which has been most studied, and for which calculations exist that our numbers can be checked against. The hexagon with Neumann conditions and other polygons, such as the octagon, will be reported in subsequent work. Consideration of symmetry classes makes computations more efficient, and gives as a byproduct the eigenvalues of a number of polygons, such as trapezoids and diamonds, which result from disecting the hexagon.

Comparisons of the hexagon results are made with Bauer and Reiss [6], who used finite-differences on an equilateral triangle mesh. Their results have been 
recomputed to correct a minor error, and they have been extended to finer mesh sizes. Comparisons are also made with Irie et al. [4], who used Fourier series, and with Jones [7], who used both finite-differences on a rectangular mesh and point-matching.

\section{THE EQUIVALENT PROBLEM}

A conformal mapping from the unit circle $C$ in the $z$-plane, $|z|<1$, to a regular $N$-gon in the $w$-plane $[8$, p. 196] is given by

$$
w=\int_{0}^{z} \frac{\mathrm{d} s}{\left(1-s^{N}\right)^{2 / N}} .
$$

The side length of the $N$-gon is

$$
S=\frac{2^{1-4 / N}}{N} \frac{\Gamma^{2}(1 / 2-1 / N)}{\Gamma(1-2 / N)} .
$$

When the eigenvalues of the $N$-gon of side $S$ are multiplied by $S^{2}$, they are converted to the eigenvalues of the unit-sided $\mathrm{N}$-gon.

The problem

$$
\Delta \Phi+\lambda \Phi=0
$$

on the $N$-gon of side $S$ is equivalent, under the correspondence $\phi(z)=\Phi(w)$, to the problem

$$
\Delta \Phi+\lambda \sigma^{2} \phi=0
$$

on $C$, with the same boundary conditions, where

$$
\sigma=\left|\frac{\mathrm{d} w}{\mathrm{~d} z}\right|=\left|1-z^{N}\right|^{-2 / N} .
$$

The quantity $\sigma^{2}$ may be thought of as a variable density on $C$.

\section{THE RAYLEIGH-RITZ UPPER BOUNDS}

The Rayleigh quotient [3] for the problem on $C$ is

$$
\frac{D(\phi, \phi)}{\iint_{C} \sigma^{2} \phi^{2} \mathrm{~d} x \mathrm{~d} y},
$$

where

$$
D(\phi, \psi) \equiv \iint_{C}\left[\frac{\partial \phi}{\partial x} \frac{\partial \psi}{\partial x}+\frac{\partial \phi}{\partial y} \frac{\partial \psi}{\partial y}\right] \mathrm{d} x \mathrm{~d} y .
$$


Upper bounds for the eigenvalues are obtained by choosing trial functions $\phi_{1}, \phi_{2}, \ldots, \phi_{M}$ and solving the $M \times M$ relative matrix eigenproblem

$$
\left[D\left(\phi_{m}, \phi_{n}\right)\right]=\mu\left[\iint_{C} \sigma^{2} \phi_{m} \phi_{n} \mathrm{~d} x \mathrm{~d} y\right] .
$$

The trial functions $\phi_{n}$ used are the eigenfunctions for (1) on $C$, which are

$$
J_{k}\left(j_{k, p} r\right)\left\{\begin{array}{l}
\cos k \theta, \\
\sin k \theta,
\end{array} \quad k=0,1,2, \ldots, p=1,2, \ldots,\right.
$$

where $j_{k, p}$ is the $p$ th root of the $k$ th Bessel function of the first type,

$$
J_{k}\left(j_{k, p}\right)=0,
$$

in the case of Dirichlet boundary conditions. For Neumann boundary conditions $j_{k, p}^{\prime}$ is used, where

$$
J_{k}^{\prime}\left(j_{k, p}^{\prime}\right)=0 .
$$

One advantage of these trial functions is that they are orthogonal in the $D$ inner product,

$$
D\left(J_{k}\left(j_{k, p} r\right) \cos k \theta, J_{l}\left(j_{l, q} r\right) \cos l \theta\right)=0,
$$

unless $k=l$ and $p=q$. (The same is true with sin functions replacing cos functions.) When $k=l$ and $p=q$, the value is $\pi j_{k, p}^{2}$ times

$$
\int_{0}^{1}\left[J_{k}\left(j_{k, p} r\right)\right]^{2} r \mathrm{~d} r=\frac{1}{2}\left[J_{k+1}\left(j_{k, p}\right)\right]^{2}
$$

for the case of Dirichlet boundary conditions, or $\pi j_{k, p}^{\prime 2}$ times

$$
\int_{0}^{1}\left[J_{k}\left(j_{k, p}^{\prime} r\right)\right]^{2} r \mathrm{~d} r=\frac{1}{2}\left[1-\left(k / j_{k, p}^{\prime}\right)^{2}\right]\left[J_{k}\left(j_{k, p}\right)\right]^{2},
$$

for Neumann boundary conditions (see, e.g., reference [19, equation 11.4.5]). When $k=0$, there is an extra factor of 2 .

Thus, the matrix on the left side of equation (4) is diagonal, so after appropriate scaling equation (4) becomes a standard eigenvalue problem for $\mu^{-1}$. The only difficult part of the analysis is the computation of the integrals on the right side of equation (4).

\section{COMPUTING THE INTEGRALS}

Using the binomial expansion,

$$
\left(1-z^{N}\right)^{-2 / N}=\sum_{k=0}^{\infty} a_{k} z^{N k}
$$


where $a_{0}=1$ and

$$
a_{k}=a_{k-1}[1-(1-2 / N) / k], \quad k=1,2, \ldots,
$$

then

$$
\begin{aligned}
\sigma^{2} & =\sum_{k, l=0}^{\infty} a_{k} a_{l} z^{N k} z^{N l}=\sum_{k, l=0}^{\infty} a_{k} a_{l} r^{N(k+l)} \mathrm{e}^{i N \theta(k-l)} \\
& =2 \sum_{n=0}^{\infty}{ }^{\prime} \cos N n \theta \sum_{l=0}^{\infty} a_{l} a_{l+n} r^{N(2 l+n)} \equiv \sum_{n=0}^{\infty} A_{n}(r) \cos N n \theta,
\end{aligned}
$$

where the prime indicates that the term for $n=0$ has a factor of $1 / 2$ on it. This defines the functions $A_{n}(r)$ for a particular value of $N$.

Thus, the integrals on the right side of equation (4) to be calculated are of the form

$$
\sum_{n=0}^{\infty} \int_{0}^{1} J_{k}\left(j_{k, p} r\right) J_{l}\left(j_{l, q} r\right) A_{n}(r) r \mathrm{~d} r \int_{-\pi}^{\pi} \cos N n \theta\left\{\begin{array}{c}
\cos k \theta \\
\sin k \theta
\end{array}\right\}\left\{\begin{array}{c}
\cos l \theta \\
\sin l \theta
\end{array}\right\} \mathrm{d} \theta .
$$

Since cos is even and $\sin$ is odd, only the integrals of cos with cos and sin with sin are non-zero. Also,

$$
\begin{aligned}
\cos N n \theta \cos k \theta \cos l \theta= & \frac{1}{4}[\cos (N n+k+l) \theta+\cos (N n+k-l) \theta \\
& +\cos (N n-k+l) \theta+\cos (N n-k-l) \theta], \\
\cos N n \theta \sin k \theta \sin l \theta= & \frac{1}{4}[-\cos (N n+k+l) \theta+\cos (N n+k-l) \theta \\
& +\cos (N n-k+l) \theta-\cos (N n-k-l) \theta] .
\end{aligned}
$$

Since

$$
\int_{-\pi}^{\pi} \cos p \theta \mathrm{d} \theta=0
$$

unless $p=0$, the $\theta$ integral vanishes unless one of

$$
N n+k+l, \quad N n+k-l, \quad N n-k+l, \quad N n-k-l
$$

is zero. This can only happen for

$$
k \equiv \pm l \quad(\bmod N) .
$$

Furthermore, at most two terms of the sum on $n$ are non-zero.

This splits the trial functions into mutually orthogonal sets called symmetry classes. The notation $\mathrm{Cl}$ will be used for the symmetry class consisting of the span of the set of functions

$$
\left\{J_{k}\left(j_{k, p} r\right) \cos k \theta: k \equiv \pm l(\bmod N), p=1,2, \ldots\right\},
$$


for $l=0,1,2, \ldots,[N / 2]$, respectively. $\mathrm{S} l$ will be used when the product is with $\sin k \theta$.

This orthogonal splitting permits the calculation of the eigenvalues of equation (4) to be efficiently done for each symmetry class separately. Furthermore, geometrical insights are gained and eigenvalues of subregions are obtained as byproducts.

The calculation of the integrals has now been reduced to determining

$$
\int_{0}^{1} J_{k}\left(j_{k, p} r\right) J_{l}\left(j_{l, q} r\right) A_{n}(r) r \mathrm{~d} r
$$

where $A_{n}(r)$ is the series in $r$ defined by equation (5), or, since the series must be truncated for computation, a large-order polynomial. The series typically converges so slowly that 4000 or so terms are needed to get satisfactory precision, so computing these integrals by a direct quadrature is very time-consuming. Hence, a recursion formula was developed for integrals of the form

$$
I_{00}(m) \equiv \int_{0}^{1} J_{k}\left(j_{k, p} r\right) J_{l}\left(j_{l, q} r\right) r^{m-1} \mathrm{~d} r
$$

The recursion is on $m$ for fixed values of $k, l, p, q$. Details are in Appendix A. This not only makes calculation of the required integrals faster, but also very accurate.

\section{THE FIXED HEXAGON}

Up to this point, the discussion has been for a general regular $N$-gon, with either fixed or free boundary conditions. Now we focus on the case of a fixed hexagon. In future work, results for free hexagons, and for other regular polygons such as the octagon will be reported.

The eigenfunctions of a region possessing symmetry can be partitioned into classes according to that symmetry. If $x=0$ is an axis of mirror symmetry for $S$, and $\Phi$ is an eigenfunction of $S$ with eigenvalue $\lambda$, so are

$$
\Phi(x, y) \pm \Phi(-x, y)
$$

if non-zero. Thus, every eigenfunction of such a region may be assumed to be either even (symmetric) or odd (antisymmetric) about the axis. The axis is a nodal line of the odd eigenfunctions and an anti-nodal line of the even eigenfunctions. Eigenfunctions in one symmetry class are orthogonal to those in the other. Regions with more than one axis of symmetry may have their eigenfunctions further partitioned.

The fixed hexagon has eight symmetry classes, C0, C1, C2, C3, S0, S1, S2, S3 as defined in the previous section and with the patterns of evenness and oddness shown in Figure 1. Dashed lines indicate a line of symmetry and solid lines indicate a line of antisymmetry. $\mathrm{C} 0$ is the class of fully symmetric functions, for which every diagonal connecting opposite vertices or the midpoints of opposite sides is a line of symmetry. S0 is the class of functions for which these diagonals are lines of 
anti-symmetry. C3 has diagonals connecting opposite vertices as lines of symmetry, and diagonals connecting midpoints of opposite sides as lines of anti-symmetry. S3 reverses these roles. $\mathrm{C} 1$ are those functions odd in $x$ and even in $y$ which are not in $\mathrm{C} 3$, while $\mathrm{S} 1$ are those functions even in $x$ and odd in $y$ which are not in S3. Finally, $\mathrm{C} 2$ are those functions even in $x$ and $y$, but not in $\mathrm{C} 0$, while $\mathrm{S} 2$ are those functions odd in $x$ and $y$, but not in $\mathrm{S} 0$.

The eigenvalues corresponding to two of these classes are known exactly. The eigenvalues of S3 are those eigenvalues of the equilateral triangle which correspond to eigenfunctions symmetric about a line from a vertex to the midpoint of the side opposite. Eigenvalues of S0 are equilateral triangle eigenvalues corresponding to eigenfunctions odd about such a line, i.e., eigenvalues of the $30^{\circ}-60^{\circ}-90^{\circ}$ triangle. These were first discovered by Lamé [10]. The equilateral triangle was re-examined by Pinsky [11], where, unfortunately, a misleading formula was given for the eigenvalues, which was also perpetuated in reference [3]. We take this opportunity to clarify this formula. The eigenvalues for a fixed equilateral triangle with unit sides are

$$
\frac{16 \pi^{2}}{9}\left(l^{2}+l m+m^{2}\right)
$$

where in S3 the integers, $l, m$ satisfy $0<l \leqslant m$, and in S0, $0<l<m$ (see Table 1 ). There is a careful discussion of the eigenfunctions and eigenvalues of the equilateral triangle by Jones [7].

The eigenvalues corresponding to $\mathrm{Cl}$ are exactly the same as the eigenvalues corresponding to $\mathrm{S} 1$. This is because an eigenfunction in $\mathrm{C} 1$ can be converted to one in S1 and vice versa. Take an eigenfunction in $\mathrm{C} 1$ and rotate it by $60^{\circ}$ positively. Also rotate it by $60^{\circ}$ negatively. Subtract the two. The result is an eigenfunction in $\mathrm{S} 1$ corresponding to the same eigenvalue. Go from $\mathrm{S} 1$ to $\mathrm{C} 1$ in a similar fashion, but adding. Likewise, the eigenvalues corresponding to $\mathrm{C} 2$ are exactly the same as those corresponding to $\mathrm{S} 2$.
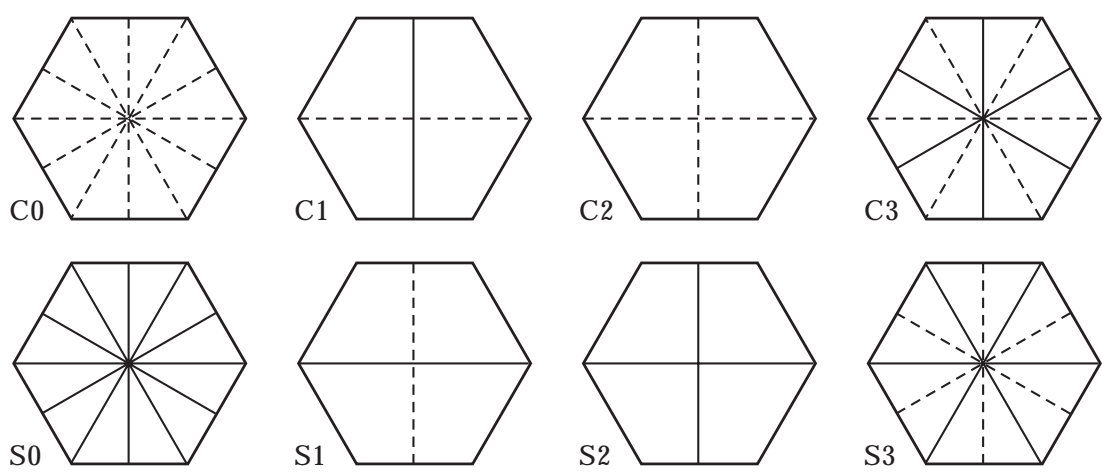

Figure 1. The eight symmetry classes of the hexagon. Dashed lines are lines of even symmetry. Solid lines are lines of odd symmetry. The eigenvalues in $\mathrm{C} 1$ and $\mathrm{S} 1$ are the same, and the eigenvalues in $\mathrm{C} 2$ and $\mathrm{S} 2$ are the same. The eigenvalues in $\mathrm{S} 0$ and $\mathrm{S} 3$ are equilateral triangle eigenvalues and are known exactly. 
TABLE 1

Eigenvalues of the unit-side equilateral triangle in S3. Those marked with an * are also in S0. The first column are the exact values to the places shown from equation (6). The second column shows the accuracy in the extrapolation formula equation (7)

\begin{tabular}{cc}
\hline \multicolumn{1}{c}{ Exact } & Extrapolation \\
\hline $52 \cdot 63789014$ & $52 \cdot 63789024$ \\
$122 \cdot 8217437^{*}$ & $122 \cdot 8217480$ \\
$210 \cdot 551561$ & $210 \cdot 551593$ \\
$228 \cdot 097524^{*}$ & $228 \cdot 097582$ \\
$333 \cdot 37330^{*}$ & $333 \cdot 37352$ \\
$368 \cdot 46523^{*}$ & $368 \cdot 46565$ \\
$473 \cdot 74101$ & $473 \cdot 74182$ \\
$491 \cdot 2870^{*}$ & $491 \cdot 2881$ \\
$543 \cdot 9249^{*}$ & $543 \cdot 9269$ \\
\hline
\end{tabular}

Therefore, computations need only be done for the symmetry classes $\mathrm{C} 0, \mathrm{C} 1$, C2, C3, since S0 and S3 are known, while S1 and S2 duplicate C1 and C2. The results of these computations are given in Tables $2-5$, where $160 \times 160$ matrices were used for each class. The trial functions used in each class were ordered by the size of the roots $j_{k, p}$ of the Bessel functions for $k$ in each class. Incidentally, no table with all of the roots needed could be found in the literature, so one was constructed, using the software package MATLAB on a PC, with which all our calculations were done. All that was required was the Bessel function routine and

TABLE 2

Eigenvalues in $\mathrm{C} 0$

\begin{tabular}{|c|c|c|c|c|}
\hline $\begin{array}{l}\text { Our upper } \\
\text { bounds }\end{array}$ & $\begin{array}{c}\text { Extrapolation } \\
\text { fd }\end{array}$ & $\begin{array}{l}\text { Jones' lower } \\
\text { bounds p-m }\end{array}$ & $\begin{array}{l}\text { Jones' } \\
\text { fd }\end{array}$ & $\begin{array}{l}\text { Jones' upper } \\
\text { bounds p-m }\end{array}$ \\
\hline $7 \cdot 155339146$ & $7 \cdot 155339122$ & $7 \cdot 1553389$ & $7 \cdot 1553415$ & $7 \cdot 1553392$ \\
\hline $37 \cdot 4913552$ & $37 \cdot 4913528$ & $37 \cdot 491264$ & $37 \cdot 4913494$ & $37 \cdot 491386$ \\
\hline $90 \cdot 058447$ & $90 \cdot 058369$ & $90 \cdot 05782$ & $90 \cdot 058354$ & $90 \cdot 05858$ \\
\hline $120 \cdot 86775$ & $120 \cdot 867572$ & $120 \cdot 86698$ & $120 \cdot 867596$ & $120 \cdot 86773$ \\
\hline $168 \cdot 82123$ & $168 \cdot 82072$ & $168 \cdot 81961$ & $168 \cdot 82065$ & $168 \cdot 82117$ \\
\hline $219 \cdot 9755$ & $219 \cdot 9732$ & $219 \cdot 9704$ & $219 \cdot 9728$ & $219 \cdot 9740$ \\
\hline $273 \cdot 7238$ & $273 \cdot 7217$ & $273 \cdot 7198$ & $273 \cdot 7211$ & $273 \cdot 7221$ \\
\hline $322 \cdot 946$ & $322 \cdot 9321$ & $322 \cdot 9256$ & $322 \cdot 9310$ & $322 \cdot 9335$ \\
\hline $360 \cdot 93952$ & $360 \cdot 93901$ & $360 \cdot 93845$ & $360 \cdot 9377$ & $360 \cdot 93867$ \\
\hline $405 \cdot 8197$ & $405 \cdot 8133$ & & & \\
\hline $464 \cdot 044$ & $463 \cdot 980$ & & & \\
\hline $538 \cdot 163$ & $538 \cdot 158$ & & & \\
\hline
\end{tabular}


TABLE 3

Eigenvalues in $\mathrm{C} 1$, which are the same as the eigenvalues in $\mathrm{S} 1$

\begin{tabular}{ccc}
\hline $\begin{array}{c}\text { Our upper } \\
\text { bounds }\end{array}$ & $\begin{array}{c}\text { Extrapolation } \\
\text { fd }\end{array}$ & $\begin{array}{c}\text { Jones' } \\
\text { fd }\end{array}$ \\
\hline $18 \cdot 1316787$ & $18 \cdot 1316778$ & $18 \cdot 1316796$ \\
$60 \cdot 105163$ & $60 \cdot 105112$ & $60 \cdot 105102$ \\
$94 \cdot 32577$ & $94 \cdot 325561$ & $94 \cdot 325547$ \\
$125 \cdot 04619$ & $125 \cdot 045468$ & $125 \cdot 045399$ \\
$152 \cdot 66460$ & $152 \cdot 664052$ & $152 \cdot 664064$ \\
$183 \cdot 4393$ & $183 \cdot 43637$ & $183 \cdot 43614$ \\
$217 \cdot 4207$ & $217 \cdot 41734$ & $217 \cdot 41709$ \\
$266 \cdot 2942$ & $266 \cdot 28054$ & $266 \cdot 28006$ \\
$282 \cdot 9213$ & $282 \cdot 90867$ & $282 \cdot 90836$ \\
$312 \cdot 7073$ & $312 \cdot 7061$ & $312 \cdot 7055$ \\
$336 \cdot 358$ & $336 \cdot 3455$ & $336 \cdot 3439$ \\
$376 \cdot 743$ & $376 \cdot 696$ & \\
$410 \cdot 909$ & $410 \cdot 844$ & \\
$426 \cdot 224$ & $426 \cdot 206$ & \\
$475 \cdot 181$ & $475 \cdot 138$ & \\
$484 \cdot 307$ & $484 \cdot 247$ & \\
$534 \cdot 91$ & $534 \cdot 59$ & \\
\hline
\end{tabular}

a slight modification of the zero finding routine included in MATLAB. In retrospect it is interesting to see how much difficulty early investigators had in finding roots of Bessel functions (see, e.g., reference [12]). The table constructed agrees with the numbers reported in references [9] and [13] for the first few Bessel functions. A copy of this table is available from the authors.

As a byproduct of consideration of the symmetry classes, eigenvalues of a number of subregions of the hexagon have been found (see Figure 2). The full hexagon has eigenvalues (including multiple eigenvalues), which are the ordered union of those in all eight classes $\mathrm{C} 0, \mathrm{C} 1, \mathrm{C} 2, \mathrm{C} 3, \mathrm{~S} 0, \mathrm{~S} 1, \mathrm{~S} 2, \mathrm{~S} 3$. If the hexagon is bisected along a line from a vertex to the opposite vertex, $60^{\circ}-120^{\circ}-120^{\circ}-60^{\circ}$ trapezoids are formed, whose eigenvalues are the ordered union of S0, S1, S2, S3. If the hexagon is bisected along a line from the midpoint of a side to the midpoint of the opposite side, $90^{\circ}-120^{\circ}-120^{\circ}-120^{\circ}-90^{\circ}$ pentagons are formed, whose eigenvalues are the ordered union of $\mathrm{S} 0, \mathrm{C} 1, \mathrm{~S} 2, \mathrm{C} 3$. Bisecting either the trapezoid or the pentagon along their axes of symmetry produces $90^{\circ}-60^{\circ}-120^{\circ}-90^{\circ}$ quadrilaterals, whose eigenvalues are the ordered union of S0 and S2, the classes common to the trapezoid and the pentagon. Trisecting the trapezoid gives equilateral triangles, whose eigenvalues are known and are the unions of S0 and S3. Trisecting the pentagon gives $60^{\circ}-90^{\circ}-120^{\circ}-90^{\circ}$ diamonds (kites), with eigenvalues the union of $\mathrm{S} 0$ and $\mathrm{C} 3$. Finally, the $30^{\circ}-60^{\circ}-90^{\circ}$ triangle is common to all and has the known eigenvalues of S0, the class common to all. 
TABLE 4

Eigenvalues in $\mathrm{C} 2$, which are the same as the eigenvalues in $\mathrm{S} 2$

\begin{tabular}{lll}
\hline $\begin{array}{c}\text { Our upper } \\
\text { bounds }\end{array}$ & $\begin{array}{c}\text { Extrapolation } \\
\text { fd }\end{array}$ & $\begin{array}{c}\text { Jones' } \\
\text { fd }\end{array}$ \\
\hline $32 \cdot 4518631$ & $32 \cdot 4518574$ & $32 \cdot 4518554$ \\
$70 \cdot 14046$ & $70 \cdot 140364$ & $70 \cdot 140357$ \\
$87 \cdot 53205$ & $87 \cdot 531946$ & $87 \cdot 531929$ \\
$145 \cdot 4836$ & $145 \cdot 48164$ & $145 \cdot 48147$ \\
$165 \cdot 5154$ & $165 \cdot 51454$ & $165 \cdot 51440$ \\
$187 \cdot 34276$ & $187 \cdot 34216$ & $187 \cdot 34191$ \\
$235 \cdot 659$ & $235 \cdot 64521$ & $235 \cdot 64491$ \\
$263 \cdot 5765$ & $263 \cdot 57572$ & $263 \cdot 57523$ \\
$274 \cdot 8664$ & $274 \cdot 8634$ & $274 \cdot 8626$ \\
$314 \cdot 535$ & $314 \cdot 5198$ & $314 \cdot 5189$ \\
$356 \cdot 84$ & $356 \cdot 78181$ & $356 \cdot 78110$ \\
$402 \cdot 216$ & $402 \cdot 208$ & \\
$420 \cdot 563$ & $420 \cdot 519$ & \\
$430 \cdot 628$ & $430 \cdot 600$ & \\
$475 \cdot 24$ & $475 \cdot 14$ & \\
$501 \cdot 87$ & $501 \cdot 71$ & \\
$541 \cdot 236$ & $541 \cdot 205$ & \\
\hline
\end{tabular}

\section{COMPARISONS WITH PREVIOUS RESULTS}

Bauer and Reiss [6] approximated the eigenvalues of a regular hexagon of unit side by using finite-differences on an equilateral triangular mesh. When the mesh width $h$ is the reciprocal of an integer, the mesh exactly fits the boundary of the hexagon. They then used Richardson extrapolation to get an improved estimate for the true eigenvalues from the finite-difference estimates for several different mesh widths (see, e.g., reference [3] for a discussion of this technique). Unfortunately, they assumed that because no interpolation is needed at the

\section{TABLE 5}

Eigenvalues in $\mathrm{C} 3$

\begin{tabular}{|c|c|c|c|c|}
\hline $\begin{array}{l}\text { Our upper } \\
\text { bounds }\end{array}$ & $\begin{array}{c}\text { Extrapolation } \\
\text { fd }\end{array}$ & $\begin{array}{l}\text { Jones' lower } \\
\text { bounds p-m }\end{array}$ & $\begin{array}{l}\text { Jones' } \\
\text { fd }\end{array}$ & $\begin{array}{l}\text { Jones' upper } \\
\text { bounds p-m }\end{array}$ \\
\hline $47 \cdot 629373$ & $47 \cdot 6293655$ & $47 \cdot 629354$ & $47 \cdot 6293634$ & $47 \cdot 629370$ \\
\hline $110 \cdot 35488$ & $110 \cdot 35474$ & $110 \cdot 35404$ & $110 \cdot 35460$ & $110 \cdot 35496$ \\
\hline $189 \cdot 5153$ & $189 \cdot 51382$ & $189 \cdot 5113$ & $189 \cdot 51349$ & $189 \cdot 5146$ \\
\hline $224 \cdot 67500$ & $224 \cdot 67482$ & $224 \cdot 67447$ & $224 \cdot 67462$ & $224 \cdot 67486$ \\
\hline $296 \cdot 7995$ & $296 \cdot 79250$ & $296 \cdot 7809$ & $296 \cdot 79192$ & $296 \cdot 7940$ \\
\hline 363.4967 & $363 \cdot 4902$ & $363 \cdot 4868$ & $363 \cdot 4891$ & $363 \cdot 4910$ \\
\hline $427 \cdot 324$ & $427 \cdot 300$ & & & \\
\hline $482 \cdot 836$ & $482 \cdot 820$ & & & \\
\hline $535 \cdot 284$ & $535 \cdot 263$ & & & \\
\hline
\end{tabular}



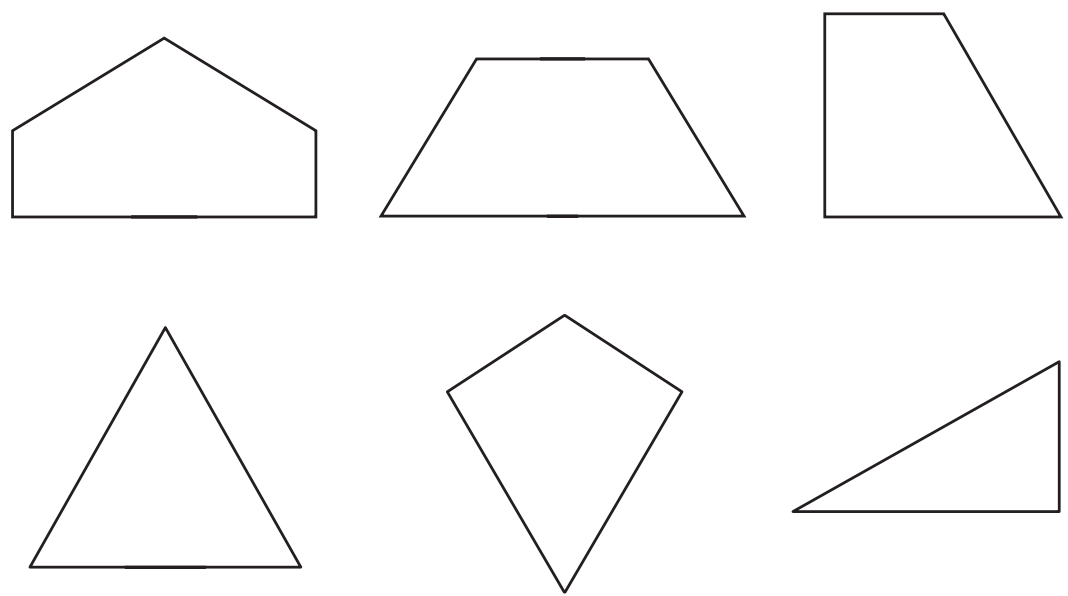

Figure 2. Polygons obtained by disecting the hexagon into symmetry classes, whose eigenvalues are also found.

boundary, the asymptotic relation between the $j$ th difference eigenvalue $\lambda_{j}(h)$ and the true $j$ th eigenvalue $\lambda_{j}$ is

$$
\lambda_{j}(h)=\lambda_{j}+a_{j} h^{2}+b_{j} h^{4}+c_{j} h^{6}+\text { higher terms. }
$$

Then by neglecting the higher terms, $\lambda_{j}$ as well as $a_{j}, b_{j}, c_{j}$ can be estimated from $\lambda_{j}(h)$ for four different values of $h$. They used $h=1 / 14,1 / 16,1 / 18,1 / 20$ for the results they reported. However, because the hexagon eigenfunctions have singularities in their derivatives at the vertices (except when they are eigenfunctions of the equilateral triangle, which are analytic in the whole plane), the correct asymptotic relation includes a cubic term and is

$$
\lambda_{j}(h)=\lambda_{j}+a_{j} h^{2}+b_{j} h^{3}+c_{j} h^{4}+\text { higher terms. }
$$

(Again, refer to reference [3].)

We have reproduced the results of Bauer and Reiss, verified that indeed this term was omitted, corrected the estimate by using the asymptotic formula (7), and extended the calculations to much finer meshes. Bauer and Reiss reported the wavenumber $k=\sqrt{ } \lambda$ for the first 21 eigenvalues to five digits past the decimal point. Their omitted term turned out to be not particularly serious, as it affected only the last digit of some of their reported results. Had they used formula (7), they would have been exact to the number of digits reported.

The sparse matrix capability of MATLAB was used to do these calculations, which were carried out for mesh widths as fine as $1 / 36$. Beyond this, roundoff seems to limit further precision. The first 104 eigenvalues of the hexagon obtained by extrapolating using equation (7) and mesh widths of $h=1 / 30,1 / 32,1 / 34,1 / 36$ are sorted into their symmetry classes and shown in Tables $1-5$. In Table 1, from the equilateral triangle eigenvalues which are known exactly, the number of correct decimal places can be seen in the extrapolated values. Presumably, adjacent extrapolated values have a similar accuracy. These extrapolated finite-difference eigenvalues are in every instance below the Ritz upper bounds of Tables 2-5, and appear to be one or two digits more accurate. 
Irie et al. [4] used a truncated Fourier series method to estimate the eigenvalues of fixed regular polygonal membranes with three to ten sides. The normalization of the eigenvalues stated in the paper is apparently not what is in fact reported. Since they compared their hexagon results with Bauer and Reiss [6], their actual normalization can be backed out of the truncated numbers they cited from Bauer and Reiss. If their hexagon numbers are multiplied by $\pi / 2$ and squared, they can be compared with our tables, and are then seen to be a bit high in the third significant figure.

Jones [7] approximated the eigenvalues of the regular hexagon by two methods, finite-differences and point-matching (or collocation). In his finite-difference scheme he used a rectangular mesh with widths $\sqrt{3} / 2 N$ and $1 / 2 N$ for integer $N$. This will also fit the boundary with no interpolation required. The largest value of $N$ he used was 100. Since his mesh widths are unequal and he did not extrapolate, the errors in his results are probably comparable to the extrapolated equilateral triangular mesh difference eigenvalues on the much coarser mesh. Jones' finite-difference results are also shown in Tables 2-5.

$\mathrm{He}$ also used point-matching on a $30^{\circ}-60^{\circ}-90^{\circ}$ triangle to approximate the eigenvalues in $\mathrm{C} 0$ and $\mathrm{C} 3$. This amounts to finding the roots $k\left(\lambda=k^{2}\right)$ of

$$
\operatorname{det}\left[J_{i}\left(k r_{j}\right) \cos i \theta_{j}\right]=0 .
$$

For $\mathrm{C} 0, i=0,6,12, \ldots, 6(N-1)$, and for $\mathrm{C} 3, i=3,9,15, \ldots, 6 N-3$. The collocation points $\left(r_{j} \cos \theta_{j}, r_{j} \sin \theta_{j}\right)$ were equally spaced along the short side of the $30^{\circ}-60^{\circ}-90^{\circ}$ triangle. When the side was divided into $N$ equal intervals and the collocation points were the centers of the intervals, Jones discovered that the roots increased with $N$. When the collocation points were placed on the ends of the intervals, the roots decreased with $N$. Jones quite reasonably inferred that the former are providing lower bounds for the eigenvalues, while the latter give upper bounds. He used $N=20$ for most results, but $N=70$ for the first C 0 eigenvalue and $N=50$ for the first $\mathrm{C} 3$ eigenvalue. Jones' point-matching results are shown in Tables 2 and 5. Jones reports $k$. We have squared his numbers to show $\lambda$.

As previously mentioned, Jones also has a very complete discussion of the exact equilateral triangle eigenfunctions and eigenvectors. He also has pictures of the level curves and nodal lines of the eigenfunctions for the first 69 eigenvalues of the hexagon.

\section{CONCLUSION}

A further example has been presented of the powerful method of using a conformal map to turn the problem of finding the eigenvalues of a homogeneous membrane of complicated shape into the problem of finding the eigenvalues of a membrane of variable density, but simple shape. This latter problem is generally easier to handle numerically. This example related regular polygons to inhomogeneous circles. The eigenvalues of the inhomogeneous circles were then estimated by the classical Rayleigh-Ritz method using the homogeneous circle eigenfunctions as trial functions. The resulting integrals required for the matrix elements were found using some Bessel function identities of some interest in 
themselves. The results of calculations for the fixed hexagon have been presented, a case where comparisons can be made with earlier investigations showing the precision of the method. As a byproduct of symmetry considerations (which simplified the calculations), the eigenvalues of subpolygons of the hexagon resulting from its disection were also obtained. Future work will cover the free hexagon and other regular polygons such as the octagon.

\section{REFERENCES}

1. J. R. KuttLer 1984 IEEE Transactions MTT 32, 348-354. A new method for calculating TE and TM cutoff frequencies of uniform waveguides with lunar or eccentric annular cross section.

2. J. R. Kuttler and V. G. Sigillito 1985 Estimating Eigenvalues with A Posteriori/A Priori Inequalities. Boston: Pittman.

3. J. R. Kuttler and V. G. Sigillito 1984 SIAM Review 26, 163-193. Eigenvalues of the Laplacian in two dimensions.

4. T. Irie, G. Yamada and K. Umesato 1981 Journal of the Acoustical Society of America 69, 1330-1336. Free vibration of regular polygonal plates with simply supported edges.

5. L. Molinari 1997 Journal of Physics A 30, 6517-6524. On the ground state of regular polygonal billiards.

6. L. BAUER and E. L. ReISS 1978 SIAM Journal of Applied Mathematics 35, 508-514. Cutoff wavenumbers and modes of hexagonal waveguides.

7. R. S. Jones 1993 Ph.D. Dissertation, Ohio State University. The one-dimensional three-body problem and selected waveguide problems: solutions of the two-dimensional Helmholtz equation.

8. Z. Nehari 1952 Conformal Mapping. New York: McGraw-Hill.

9. M. Abramowitz and I. A. Stegun 1964 Handbook of Mathematical Functions. Washington, DC: National Bureau of Standards.

10. M. G. Lamé 1866 Leçons sur la Théorie Mathématique de l'Élasticité des Corps Solides. Paris: Gauthiers-Villars.

11. M. A. PINSKY 1980 SIAM Journal of Mathematical Analysis 11, 819-827. The eigenvalues of an equilateral triangle.

12. W. G. Bickley and J. C. P. Miller 1945 Philosophical Magazine 36, 121-133. Notes on the evaluation of zeros and turning points of Bessel functions.

13. H. GERBER 1964 Mathematics of Computation. 18, 319-322. First one hundred zeros of $J_{0}(x)$ accurate to 19 significant digits.

14. Y. L. LuKe 1962 Integrals of Bessel Functions. New York: McGraw-Hill.

\section{APPENDIX A: THE BESSEL FUNCTION INTEGRAL ITERATION}

For fixed integers $m$ and $n$, and fixed roots $\alpha$ of $J_{m}$ and $\beta$ of $J_{n}$ such that

$$
J_{m}(\alpha)=0, \quad J_{n}(\beta)=0,
$$

define, for $k=1,2, \ldots$,

$$
I_{00}(k) \equiv \int_{0}^{1} J_{m}(\alpha x) J_{n}(\beta x) x^{k-1} \mathrm{~d} x, \quad I_{10}(k) \equiv \int_{0}^{1} \frac{\mathrm{d}}{\mathrm{d} x}\left[J_{m}(\alpha x)\right] J_{n}(\beta x) x^{k} \mathrm{~d} x,
$$




$$
\begin{gathered}
I_{01}(k) \equiv \int_{0}^{1} J_{m}(\alpha x) \frac{\mathrm{d}}{\mathrm{d} x}\left[J_{n}(\beta x)\right] x^{k} \mathrm{~d} x, \\
I_{11}(k) \equiv \int_{0}^{1} \frac{\mathrm{d}}{\mathrm{d} x}\left[J_{m}(\alpha x)\right] \frac{\mathrm{d}}{\mathrm{d} x}\left[J_{n}(\beta x) x^{k+1} \mathrm{~d} x .\right.
\end{gathered}
$$

If one starts with the expression

$$
\int_{0}^{1} \frac{\mathrm{d}}{\mathrm{d} x}\left[J_{m}(\alpha x) J_{n}(\beta x)\right] x^{k} \mathrm{~d} x,
$$

on the one hand one can differentiate directly, and on the other hand one can integrate by parts, using the fact that $\alpha$ and $\beta$ are roots, to get the identity

$$
I_{10}(k)+I_{01}(k)+k I_{00}(k)=0 .
$$

One can do a similar thing, starting with

$$
\int_{0}^{1} \frac{\mathrm{d}}{\mathrm{d} x}\left\{\frac{\mathrm{d}}{\mathrm{d} x}\left[J_{m}(\alpha x)\right] J_{n}(\beta x)\right\} x^{k+1} \mathrm{~d} x,
$$

and using the Bessel equation

$$
\frac{\mathrm{d}^{2}}{\mathrm{~d} x^{2}} J_{m}(\alpha x)+\frac{1}{x} \frac{\mathrm{d}}{\mathrm{d} x} J_{m}(\alpha x)+\left(\alpha^{2}-\frac{m^{2}}{x^{2}}\right) J_{m}(\alpha x)=0
$$

to get (after some rearranging)

$$
m^{2} I_{00}(k)+k I_{10}(k)+I_{11}(k)=\alpha^{2} I_{00}(k+2) .
$$

Similarly, interchanging $m$ and $n, \alpha$ and $\beta$,

$$
n^{2} I_{00}(k)+k I_{01}(k)+I_{11}(k)=\beta^{2} I_{00}(k+2) .
$$

Finally, starting with

$$
\int_{0}^{1} \frac{\mathrm{d}}{\mathrm{d} x}\left\{\frac{\mathrm{d}}{\mathrm{d} x}\left[J_{m}(\alpha x)\right] \frac{\mathrm{d}}{\mathrm{d} x}\left[J_{n}(\beta x)\right]\right\} x^{k+2} \mathrm{~d} x,
$$

leads to

$$
n^{2} I_{10}(k)+m^{2} I_{01}(k)+k I_{11}(k)=\beta^{2} I_{10}(k+2)+\alpha^{2} I_{01}(k+2)+\alpha \beta J_{m}^{\prime}(\alpha) J_{n}^{\prime}(\beta) .
$$

We have not been able to find these identities in the Bessel function literature for general $m$ and $n$. The special cases of 0 and 1 are on pp. 262-263 of reference [14]. 
Equations (A1)-(A4) may be used for either an upward or a downward iteration. Both iterations become unstable at small values of $k$ (depending on $m$, $n, \alpha$ and $\beta$ ). The downward iteration is used here. Several remarks may be made on the iteration. For large values of $k$, the downward iteration is very stable, so the initial values of $I$ can be anything (we just use zero), and after a few iterations the values will be correct to full precision. We are only interested in $I_{00}$, so equation (A4) can be used to eliminate $I_{11}$ from equations (A2) and (A3), and the values of $I_{01}$ and $I_{10}$ may be overwritten at each step. Thus, define

$$
R S=\beta^{2} I_{10}+\alpha^{2} I_{01}+\alpha \beta J_{m}^{\prime}(\alpha) J_{n}^{\prime}(\beta),
$$

where $I_{10}$ and $I_{01}$ are the values at $k+2$. Then the values of $I_{10}$ and $I_{01}$ at $k$ are given by

$$
\begin{aligned}
{\left[\begin{array}{c}
I_{10} \\
I_{01}
\end{array}\right]=} & \frac{1}{\left(k^{2}-m^{2}-n^{2}\right)^{2}-4 m^{2} n^{2}}\left[\begin{array}{cc}
k^{2}-m^{2}-n^{2} & 2 m^{2} \\
2 n^{2} & k^{2}-m^{2}-n^{2}
\end{array}\right] \\
& \times\left[\begin{array}{l}
\alpha^{2} k I_{00}(k+2)-R S \\
\beta^{2} k I_{00}(k+2)-R S
\end{array}\right],
\end{aligned}
$$

and then

$$
I_{00}(k)=-\frac{1}{k}\left(I_{10}+I_{01}\right)
$$

Further, the values of $I_{00}(k)$ are only needed for $k$ even, so the iteration can be specialized to this case. Also, in the particular situation when $\alpha=\beta$ (implying that $m=n$ also), the iteration simplifies.

To determine the minimum value $k_{M}$ for which the iteration is stable, let the error $E_{00}(k)$ be the difference between the true value of $I_{00}(k)$ and the calculated value, and similarly for $E_{10}$ and $E_{01}$. Then the errors satisfy the same equations (A1)-(A4), except the term $\alpha \beta J_{m}^{\prime}(\alpha) J_{n}^{\prime}(\beta)$ is not present. To simplify the error analysis, let

$$
\varepsilon=\max \left(\left|E_{10}\right|,\left|E_{01}\right|\right)
$$

Now

$$
E_{00}(k+2)=-\frac{1}{k+2}\left(E_{10}(k+2)+E_{01}(k+2)\right),
$$

from which follows the simple estimate

$$
\left|E_{00}(k+2)\right| \leqslant \frac{2}{k} \varepsilon
$$


which put into (A5), when $k^{2} \geqslant m^{2}+n^{2}$, gives

$$
\begin{aligned}
{\left[\begin{array}{l}
E_{10}(k) \\
E_{01}(k)
\end{array}\right] \leqslant } & \frac{1}{\left(k^{2}-m^{2}-n^{2}\right)^{2}-4 m^{2} n^{2}}\left[\begin{array}{cc}
k^{2}-m^{2}-n^{2} & 2 m^{2} \\
2 n^{2} & k^{2}-m^{2}-n^{2}
\end{array}\right] \\
& \times\left[\begin{array}{c}
\left(3 \alpha^{2}+\beta^{2}\right) \varepsilon \\
\left(\alpha^{2}+3 \beta^{2}\right) \varepsilon
\end{array}\right] .
\end{aligned}
$$

Thus, a sufficient condition for the ratio of the new value of $\varepsilon$ to the old one to be no larger than one is

$$
\begin{aligned}
& \frac{\left(k^{2}-m^{2}-n^{2}\right)\left(3 \alpha^{2}+\beta^{2}\right)+2 m^{2}\left(\alpha^{2}+3 \beta^{2}\right)}{\left(k^{2}-m^{2}-n^{2}\right)^{2}-4 m^{2} n^{2}} \leqslant 1, \\
& \frac{\left(k^{2}-m^{2}-n^{2}\right)\left(\alpha^{2}+3 \beta^{2}\right)+2 n^{2}\left(3 \alpha^{2}+\beta^{2}\right)}{\left(k^{2}-m^{2}-n^{2}\right)^{2}-4 m^{2} n^{2}} \leqslant 1 .
\end{aligned}
$$

These are quadratic inequalities in $k^{2}$, and solving gives

$$
k^{2} \geqslant m^{2}+n^{2}+\frac{1}{2}\left(3 \alpha^{2}+\beta^{2}\right)+\sqrt{4 m^{2} n^{2}+\frac{1}{4}\left(3 \alpha^{2}+\beta^{2}\right)^{2}+2 m^{2}\left(\alpha^{2}+3 \beta^{2}\right)},
$$

in the first case, with a similar inequality with $m$ and $n, \alpha$ and $\beta$ reversed in the second case. Hence, $k_{M}^{2}$ is the maximum of these expressions.

Now the integrals we want to compute are of the form

$$
\int_{0}^{1} J_{m}(\alpha x) J_{n}(\beta x) \sum_{k=1}^{L} p(k) x^{k-1} \mathrm{~d} x
$$

which we write as

$$
\int_{0}^{1} J_{m}(\alpha x) J_{n}(\beta x) \sum_{k=1}^{k_{M}-1} p(k) x^{k-1} \mathrm{~d} x+\sum_{k=k_{M}}^{L} p(k) I_{00}(k),
$$

and we know $I_{00}(k)$ for $k$ down to $k_{M}$ from the recursion above, while we compute the first term using a quadrature formula. 